\title{
Good Literacy to Enhance Response in Diabetes Mellitus (GLITTER-DM)
}

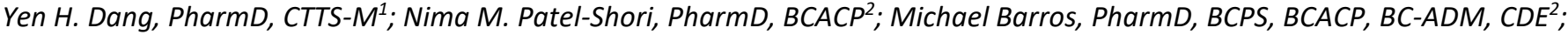 \\ Daohai $Y u, P h D, M S^{3}$ \\ ${ }^{1}$ University of Maryland Eastern Shore, School of Pharmacy and Health Professions, Department of Pharmacy Practice \\ ${ }^{2}$ Temple University, School of Pharmacy, Department of Pharmacy Practice \\ ${ }^{3}$ Temple University, School of Medicine, Department of Clinical Sciences
}

\begin{abstract}
Objective: The purpose of this study is to determine the effectiveness of individualized communication strategies and self-management action plans to improve A1c control at 3 months in patients with low health literacy.

Methods: A prospective, open-labeled, pilot study was conducted on 23 patients with diabetes mellitus in a pharmacist-led ambulatory care clinic. Patients who had a Rapid Estimate of Adult Literacy in Medicine-Revised (REALM-R) score of 6 or less and an A1c greater than $7 \%$ upon study entry were included. The first group received the teach-back method, personalized actions, and follow-up phone calls to assess comprehension $(N=12)$. The second group was assigned to usual care $(N=11)$.

Results: Patients receiving literacy-appropriate interventions had greater A1c percent reduction (A1c difference of -2.0 \pm 1.3 vs $-1.0 \pm$ 2.2; $P=0.02$ ) and less hyperglycemic events per week (0.1 vs. 2.1; $P=0.04)$. There were no differences in the number of hypoglycemic events, testing frequency, medication-adherence rates, or hospitalizations and emergency room visits related to diabetes.

Conclusion: Literacy-appropriate methods such as the teach-back method, personalized action plans, and telephone follow-ups may improve glycemic control in low health literate patients with diabetes.

Practice Implications and Innovations: The findings from this study suggest that pharmacists may improve diabetes outcomes when managing patients who possess low health literacy using simplified teaching methods.
\end{abstract}

Key words: health literacy, diabetes, education, teach-back, action plans

\section{Introduction}

Diabetes is a chronic disease that currently affects 30.3 million Americans and has resulted in substantial morbidity, mortality, and financial burden. ${ }^{1}$ Among these, it is estimated that $15-40 \%$ of all patients with diabetes possess low health literacy. ${ }^{2}$ Health literacy is defined as the "degree in which individuals have the capacity to obtain, process, and understand basic health information and services needed to make appropriate health decisions". ${ }^{3}$ Health numeracy is a component of health literacy and involves the understanding of numbers in disease state management. Daily diabetes activities rely on having adequate numeracy skills to carry out self-care tasks including carbohydrate counting, insulin titration, and blood glucose monitoring. Many aspects of diabetes care require adequate health literacy including lifestyle management, appropriate medication usage, self-testing procedures, and selfmanagement of hyperglycemia and hypoglycemia. Studies show that patients with poor health literacy have worse clinical

Corresponding author: Yen H. Dang, PharmD, CTTS-M Associate Professor of Pharmacy Practice University of Maryland Eastern Shore School of Pharmacy and Health Professions One College Backbone Road 210 Somerset Hall,Princess Anne, MD 21853

Phone: 410-651-8169; Fax: 410-651-8394

E-mail: ydang@umes.edu outcomes. ${ }^{4}$ For every one-point decrease in the Short Test of Functional Health Literacy Assessment (s-TOFHLA) score, a $0.02 \%$ rise in the $A 1 c$ was observed. Patients with diabetes who have limited health literacy also have poorer disease state knowledge, lower self-confidence in diabetes management, and difficulty understanding and adhering to medication instruction. $^{5}$

While there is consensus in the definitions for health literacy and standardized health literacy measurement instruments, gaps in the literature currently remain on the association of health literacy to health outcomes in patients with diabetes. Empiric research contains contradictory results about the association of health literacy to self-care, adherence, and glycemic control. ${ }^{6,7}$ Additionally, it is unclear how health literacy is associated with self-efficacy, which is the relationship between knowledge and behavior, and how health professionals' communication strategies can impact patient understanding. ${ }^{6,7}$ Sayah et al. ${ }^{8}$ revealed that only $11 \%$ of all communication loops in a diabetes primary care center were closed where health information was either clarified, repeated, or checked for understanding. Medical jargon and mismatched language were used often in encounters in patients with low health literacy. ${ }^{8}$ Finally, the cost-effectiveness of interventions that improve health literacy are unknown. Limited health literacy in patients with type 2 diabetes adds an additional 3$5 \%$ to total healthcare costs in the United States every year. ${ }^{7}$ Best practices which are simplistic to carry out in a primary care 
setting for patients with diabetes so that these patients may improve their glycemic control, frequency of testing, and medication adherence to ultimately drive down healthcare costs is largely needed. This study aims to explore the relationship between low health literacy and diabetes outcomes using cost-effective interventions by pharmacy professionals.

The use of the teach-back method and personalized action plans are simple, cost-effective techniques that have been shown to be successful in changing health behavior. ${ }^{9}$ The teachback method is a framework for assessing and enhancing patient understanding by closing the communication loop. By allowing patients to explain in their own words medical information, the teach-back method improves patient-provider communication, promotes medication adherence, and increases patient safety. ${ }^{9}$ Personalized action plans revolve around the socio-behavioral model of care where patients have an active voice in the decision-making process. ${ }^{9}$ In this patientcentered approach, the patient can negotiate their behavior during the information exchange to allow for realistic, successful treatment regimens. ${ }^{9}$ Both methods allow the patient to be actively engaged in their own care and accountable for their own health. Few studies thus far have assessed the impact of a pharmacist-driven program using these combined methods to improve glycemic control in patients with low health literacy.

The purpose of this study is to determine the effectiveness of individualized communication strategies and self-management action plans to improve A1c control at 3 months in patients with low health literacy. We hypothesize that patients who receive targeted counseling sessions and individualized goal-setting plans will have achieved better glycemic control compared to those who receive standard of care.

\section{Research Design}

A prospective, open-labeled, pilot study was conducted in Medicine Group Practice (MGP) clinic patients at a tertiary-care, teaching hospital. Patients were seen for scheduled study visits at one to four week intervals over a planned period of 3 months. An institutional review board at the site approved the protocol, and patients provided written informed consent.

Patients were eligible to participate if they were at least 18 years of age with a diagnosis of type 1 or type 2 diabetes and possessed low health literacy, defined as a Rapid Estimate of Adult Literacy in Medicine- Revised (REALM-R) score of 6 or less. The REALM- $R$ is a validated health literacy instrument used to assess a person's ability to read and pronounce 11 common medical words. ${ }^{10}$ The test assesses how well patients read words that are common to the primary care setting and are expected to understand when interacting with their provider. While there are many standardized health literacy tests available (e.g. s-TOFHLA, Newest Vital Sign [NVS]), the REALM$\mathrm{R}$ was chosen for this study due to its rapid administration and ease of scoring. Research has shown that scores of 6 or less out of a total of 8 points identify patients at risk for low health literacy. ${ }^{10}$ In addition, patients also needed to have an A1c greater than $7 \%$ upon study entry. Patients with a diagnosis of stroke in the past 3 months, dementia, Alzheimer's disease, receiving pharmacotherapy for Alzheimer's or dementia, such as acetylcholinesterase inhibitors or memantine, were excluded. In addition, non-English speakers, patients with visual impairment, and patients without access to a telephone were not eligible for this study.

\section{Methodology}

All eligible patient underwent block randomization into the interventional or usual care arm. Both groups met with pharmacists in a collaborative-practice run clinic for medication management and diabetes education. During enrollment, the REALM-R and Diabetic Numeracy Test-5 (DNT-5) was administered to all subjects. The DNT- 5 is a standardized assessment test consisting of mathematical calculations and real-world applications to determine numeracy skills in patients with diabetes. ${ }^{6}$ This abbreviated five-question DNT-5 was also chosen for its quick administration and scoring over the original test, the 43-question Diabetic Numeracy Test (DNT). Subjects who score 3 or less on this test out of a total 5 points are more likely to possess low numeracy skills. Both instruments are available on the public domain.

Patients in the interventional arm received the teach-back method and action plans at every clinic visit to re-assess their knowledge (Table 1). The teach-back method involved asking the patient to explain or demonstrate what they have been told in order to confirm understanding. An action plan consisting of one to three goals was also created for diabetes management at the end of the visit. Action plans were created through a shared decision making approach between the provider and patient working collaboratively to allow for patient empowerment. The patient rated their confidence of achieving the action plan on a Likert scale of 1 (not sure) to 10 (very sure). Action plans with a score of 6 or less were modified so that the patient was more comfortable with completing the goal. ${ }^{9}$

In addition, follow-up phone calls were conducted every 2-4 weeks at the halfway point in-between visits to assess comprehension and for further education reinforcement. The teach-back method was also performed during these phone sessions, and action plans were also allowed to be modified if the score was 6 or less. Patients in the control arm received usual care only, without the use of the teach-back method, action plans, or any telephone follow-ups. In this practitionercentered model, even though information was given during appointments, patients were not assessed for their understanding through the teach-back method to close the communication loop, and treatment decisions were made solely by the pharmacist without assessing the patient's confidence level to carry out the plan. 
A pharmacist with specialized knowledge and training in diabetes carried out all protocol procedures including education, medication titration, data recording, and monitoring. The same pharmacist personnel interacted with all study participants to ensure continuity and reliability. Both groups were managed under the clinic's collaborative practice agreement treatment protocol for diabetes, and standardized insulin titration procedures including Treat-to-Target and the rule of 1800 were used to adjust insulin dosages. ${ }^{11,12}$ The Pharmacist's Patient Care Process was followed through the collaborative practice agreement to deliver evidence-based care to all participants through data collection, assessment, planning, implementation, and evaluation. ${ }^{13}$

At every study visit, blood glucose values were obtained by the pharmacist using a point-of-care glucometer. The frequency of hypoglycemia or hyperglycemia and frequency of testing were screened every encounter by patient-reported questionnaires and through the patient's self-monitoring blood glucose (SMBG) logs. In addition, hospitalization and emergency room visits related to diabetes were measured by patient report and confirmed with medical records. Total adherence rate for the study duration was verified by pharmacy refill records through the software program PrescribeWellness. A1c samples were taken by laboratory venous blood tests at baseline and upon study termination as per standard of care practice guidelines.

\section{Study Endpoints}

The primary endpoint was the mean change in A1c value at 3 months. This value was represented in one of two ways; either as the difference between the initial and final A1c, or shown as a percent reduction by the above value divided by the initial A1C multiplied by 100 percent. For example, if a subject has a baseline A1c of $8 \%$ (183 mg/dL, $10.2 \mathrm{mmol} / \mathrm{L})$ upon study entry and the A1c decreases to $7.1 \%(157 \mathrm{mg} / \mathrm{dL}, 8.7 \mathrm{mmol} / \mathrm{L})$ at the 3 month period, then the difference in $\mathrm{A} 1 \mathrm{c}$ is $-0.9 \%$ and the percent reduction is $-11.25 \%$.

Secondary endpoints included the number of blood glucose values obtained during clinical visits that were less than 70 $\mathrm{mg} / \mathrm{dL}$ (3.88 mmol/L) or greater than $180 \mathrm{mg} / \mathrm{dL}$ ( $9.99 \mathrm{mmol} / \mathrm{L})$. The number of patients reaching an A1c of less than $7 \%$ and the change in frequency of glucose testing from baseline were assessed. The frequency of hypoglycemia, defined as a glucose level less than $70 \mathrm{mg} / \mathrm{dL}(3.88 \mathrm{mmol} / \mathrm{L})$ or symptoms of low blood sugar such as dizziness, sweating, shaking or trembling, or hyperglycemia, defined as a glucose level of greater than $300 \mathrm{mg} / \mathrm{dL}$ (16.65 $\mathrm{mmol} / \mathrm{L})$ or symptoms of high blood sugar such as extreme thirst, frequent urination, drinking or eating episodes, were also recorded. Other secondary endpoints include the number of hospitalizations or emergency room visits for diabetes-related complications and medication adherence rates over the course of 3 months.

\section{Statistical Analysis}

A total of 40 patients were needed to obtain a statistical power of 80 percent to detect a $1 \%$ difference in $A 1 \mathrm{c}$ values between the two treatment arms. However, to account for drop-outs, a goal of 50 patients for recruitment was selected. All P-values were 2 -sided and P-values $\leq 0.05$ were considered statistically significant. Continuous variables were presented as means with standard deviations, and P-values for continuous categorical data were computed with the use of a nonparametric Wilcoxon test. Fisher's Exact Test was used for dichotomous categorical data. Treatment comparisons were performed with the use of a per-protocol analysis. Data was analyzed using SPSS ${ }^{\circledR}$ and Minitab ${ }^{\oplus}$

\section{Results}

A total of 25 patients underwent randomization during the 3 month enrollment phase (Figure 1). Of these, 13 patients were assigned to receive health-literacy appropriate interventions and 12 patients were assigned to receive usual care. At the end of the study period, 2 patients (15.38\%) were lost to follow-up in the interventional arm, leaving a total of 11 patients in the treatment arm and 12 patients in the usual care arm to be included in the primary analysis. Baseline demographics are shown in Table 2 . The study sample was comprised of a middleaged population that was predominantly women $(60.87 \%)$ and African-American (91.30\%). The majority of patients possessed a high-school education (56.52\%) or lower and had type 2 diabetes (95.65\%). The two groups were well matched for baseline characteristics except for duration of diabetes $(P=$ 0.01). In regards to the type of diabetes regimen (Table 3 ), the majority of patients were on an insulin-oral combination and the usual care arm had a higher total usage of insulin per kilogram of body weight compared to intervention arm (0.96 \pm 0.78 vs. $0.41 \pm 0.35$, respectively, $P=0.05$ ). Scores of 6 or less on the REALM-R and 3 or less on the DNT-5 indicate low literacy and numeracy. The average REALM-R score of 2 and 4.1 in usual care and intervention, respectively, as well as a mean DNT-5 score of 1.3 in both arms reveals a population that had both poor health literacy and numeracy. There were also no differences in baseline $A 1 c$ in both treatment arms $(9.3 \%$ in usual care arm vs. 9.7\% in interventional arm, $P=0.47)$. Patients in the interventional arm created an average of two action plans per visit.

At the end of three months, most patients were still on an insulin-oral regimen and there were more patients on an insulin-only regimen in the usual care arm $(P=0.04)$. There were no differences in the total units of insulin per kilogram of body weight between the two arms at the end of the study $(0.84 \pm 0.53$ vs. $0.48 \pm 0.31, P=0.12)$. While average A1c value at study termination was comparable between the groups (Figure 2), the treatment arm achieved a greater reduction in the primary endpoint (Table 4). The A1c difference was a mean reduction of only $1 \%$ in the usual care arm compared to a $2 \%$ reduction in the treatment $\operatorname{arm}(P=0.02)$. The intervention team also had a greater mean A1c percent reduction of $20.3 \%$ 
versus a $9.1 \%$ decrease in the usual care arm $(P=0.04)$. There were no differences in the number of patients who reached a A1c of less than $7 \%(154 \mathrm{mg} / \mathrm{dL}, 8.7 \mathrm{mmol} / \mathrm{L})$ or the number of blood glucose values in clinic that were less than $70 \mathrm{mg} / \mathrm{dL}(3.88$ $\mathrm{mmol} / \mathrm{L}$ ) or greater than $180 \mathrm{mg} / \mathrm{dL}(9.99 \mathrm{mmol} / \mathrm{L})$. While there was no difference between the number of hypoglycemic events, patients in the treatment arm experienced less hyperglycemic events per week ( 0.1 vs. $2.1 ; P=0.04)$. Finally, there were no statistical differences between the number of hospitalizations or emergency room visits related to diabetes, testing frequency, and medication adherence rates.

\section{Discussion}

Many misconceptions may arise in patients with low literacy when controlling and managing their diabetes which lead to complications. In a study where participants had below a 6th grade reading level with an $A 1 \mathrm{c}$ of greater than $8 \%(183 \mathrm{mg} / \mathrm{dL}$, $10.2 \mathrm{mmol} / \mathrm{L}$ ), $61 \%$ believed they were managing their diabetes well and correspondingly did not see their physician. ${ }^{14}$ Patients who believe that they have firm control of their diabetes are less likely to seek medical assistance and make changes in their regimen. Additionally, a study conducted by Fan et al. ${ }^{15}$ showed low-income, African-Americans with low health literacy and an education level of high school or less had higher rates of medication non-adherence. The patient demographics in Fan et al. ${ }^{15}$ were similar to the participants in the GLITTER-DM study with equal rates of non-adherence. Patients with diabetes who possess low health literacy may have trouble following prescription medication instructions including indication, dosage, frequency of administration, and special instructions. ${ }^{16}$ There is also an association where patients with diabetes who have lower literacy levels will have worse communication quality, higher levels of mistrust, and less engagement with medical providers. ${ }^{17,18}$ Not only does this impact their medical care, but this population has worse self-care routines including lower rates of physical fitness and healthy eating habits. ${ }^{18}$ Similarly, patients in the GLITTER-DM study assigned to usual care had difficulty understanding medical information, performing self-care behaviors, and maintaining glycemic control. The complexities of literacy affect all aspects of a patient's disease-state management and have the potential for serious consequences.

The results of this pilot study suggests that closing the communication loop by using simple literacy-appropriate intervention strategies, such as the teach-back method and action plans, as well as telephone follow-up calls to reinforce patient understanding are effective methods of improving diabetes control. Similarly, Wallace et al. ${ }^{19}$ also performed these interventions in a sample where $28.8 \%$ of the subjects had inadequate health literacy and found improvements in selfefficacy, diabetes-related distress, and knowledge $(P<0.001)$. Another study by Rothman et al. ${ }^{20}$ showed that the teach-back method and simplified medical jargon resulted in A1c reductions for patients with low literacy (adjusted difference $1.4 \%, \mathrm{P}<0.001$ ) and allowed for more subjects to obtain an A1C of $\leq 7 \%$ (odds ratio $4.6, P=0.02$ ). While this study did not reveal changes in endpoints other than A1c and hyperglycemia, other trials have shown such a benefit. For instance, Kim et al. ${ }^{21}$ showed that diabetes education classes improved self-blood glucose monitoring $(P=0.002)$. This study was not powered to detect a result in the secondary endpoints and the study size may have been too small to detect a difference. The mean adherence rate, $87.7 \%$ in interventional arm versus $78.0 \%$ in usual care arm, respectively, may have been significant if the sample size was larger.

Other novel methods created to modify diabetes outcomes in patients with low health literacy have been explored. The Partnership to Improve Diabetes Education (PRIDE) toolkit contains a series of diabetes self-management modules in English and Spanish created at a 5th grade reading level. ${ }^{22}$ The toolkit contains diabetes information and shared goal setting activities for patients with low health literacy and numeracy. ${ }^{22}$ Lay health trainers, the use of peer or lay educators in the community, have also been used to improve self-management, quality of life, mental health, and illness perception in patients with low literacy and poorly controlled diabetes. ${ }^{23} \mathrm{~A}$ study by Sugita et al. ${ }^{24}$ used health-literate text messages to improve medication adherence for patients with diabetes. While these approaches are more complex, all have proved to be effective interventions on diabetes self-care and knowledge.

\section{Strengths and Limitations}

The strengths of this study were that it was a randomized, controlled trial and that the inclusion criteria was limited to low literacy patients including those with lower socioeconomic status or education, minorities, and seniors. The primary and secondary endpoints assessed validated surrogate markers for diabetes and the interventions were simple as well as achievable in an ambulatory care clinic setting. Additionally, the intervention group spent an average length of 25 minutes in the clinic versus the usual care arm of only 20 minutes. Thus, these literacy-appropriate interventions could be replicated in a fastpaced ambulatory care practice setting with minimal time restrictions.

Limitations of the pilot study included the small sample size and short follow-up time. Due to the small study sample, the results were also non-parametric. Numerous health literacy assessment instruments are available but the REALM-R was chosen for ease in administration and scoring. However, limitations of the instrument include that it is a word recognition test, not a reading comprehension tool. At baseline, while the mean REALM- $R$ values between the two arms did not reach statistical significance, slightly higher scores in health literacy may predispose patients in the interventional arm to have better knowledge on diabetes issues and be more comfortable with receiving medication instruction. However, since the inclusion criteria focused primarily on low-literacy populations, this difference may not have been clinically relevant and the interventions used to deal with this 
population, regardless of REALM-R score, may be universal for all. Finally, patients in the usual care arm had a longer duration of diabetes since they were older and were on higher total doses of insulin at baseline, which may have influenced the study results. For example, while these patients may have been exposed to a longer period of diabetes education from the time of diagnosis, the course of diabetes may be more complicated and difficult to treat.

\section{Conclusion}

To improve glycemic control, health literacy levels need to be considered when formulating educational strategies to improve health outcomes. The teach-back method, personalized action plans, and phone follow-ups may lower A1c and reduce hyperglycemic events in low health literate patients with diabetes. Additional studies with larger sample sizes should be done to determine if these methods are effective in patients with diabetes along with other chronic care diseases requiring self-management.

\section{Practice Implications and Innovations}

The findings from this study suggest that pharmacists can improve control of diabetes in those with low health literacy and numeracy using simplified teaching methods. The teachback method to verify patient understanding, patient-provider created action plans to improve self-management, and followup phone calls to reinforce information are all cost-effective methods for diabetes education. This combined treatment approach may lead to reductions in A1c as well as hyperglycemic symptoms. While these interventions were performed at a pharmacy-led collaborative practice clinic, the methods may be applied to patients with low health literacy in all outpatient settings to improve diabetes outcomes. The study adds to the evidence that simple methods addressing the obstacles of low health literacy may be utilized to improve outcomes in patients with diabetes.

Acknowledgements: The authors would like to thank Xing Jin, Temple University School of Pharmacy, PharmD, for his assistance with data collection in this study.

Financial Disclosure: This research did not receive any specific grant from funding agencies in the public, commercial, or notfor-profit sectors.

Conflicts of Interest: We declare no conflicts of interest or financial interests that the authors or members of their immediate families have in any product or service discussed in the manuscript, including grants (pending or received), employment, gifts, stock holdings or options, honoraria, consultancies, expert testimony, patents and royalties.

Treatment of Human Subjects: IRB review/approval required and obtained

Dual Publication: The results/data/figures in this manuscript have not been published elsewhere nor are they under consideration in another journal.

\section{References}

1. Centers for Disease Control and Prevention. National diabetes fact sheet: national estimates and general information on diabetes and prediabetes in the United States, 2017. Atlanta, GA: U.S. Department of Health and Human Services, Centers for Disease Control and Prevention, 2017.

https://www.cdc.gov/diabetes/pdfs/data/statistics/n ational-diabetes-statistics-report.pdf. Accessed November 21, 2019.

2. Cavanaugh KL. Health literacy in diabetes care: explanation, evidence and equipment. Diabetes Manag. 2011;1:191-199. doi: 10.2217/dmt.11.5.

3. Ratzan SC, Parker RM. Introduction. In: Selden CR, Zorn M, Ratzan SC, Parker RM, editors. In National Library of Medicine current bibliographies in medicine: Health literacy. Bethesda, MD: National Institutes of Health; 2000. (NLM Pub. No. CBM 20001).

4. Schillinger D, Grumbach K, Piette J, et al. Association of health literacy with diabetes outcomes. JAMA. 2002;288:475-482. doi: 10.1001/jama.288.4.475.

5. White RO, Wolff K, Cavanaugh KL, et al. Addressing health literacy and numeracy to improve diabetes education and care. Diabetes Spectr. 2010; 23:238243. doi: 10.2337/diaspect.23.4.238.

6. Al Sayah F, Williams B, Johnson JA. Measuring health literacy in individuals with diabetes: A systematic review and evaluation of available measures. Health Educ Behav. 2013; 40:42-55. doi: 10.1177/1090198111436341.

7. Caruso R, Magon A, Baroni I, et al. Health literacy in type 2 diabetes patients: a systematic review of systematic reviews. Acta Diabetol. 2018; 55:1-12. doi: 10.1007/s00592-017-1071-1.

8. Al Sayah F, Williams B, Pederson JL, et al. Health literacy and nurses' communication with type 2 diabetes patients in primary care settings. Nurs Res. 2014; 63(6): 408-417. doi: 10.1097/NNR.0000000000000055.

9. Agency for Healthcare Research and Quality. AHRQ Health Literacy Universal Precautions Toolkit. Rockville, MD: Agency for Healthcare Research and Quality, US Department of Health and Human Services; 2018.

http://www.ahrq.gov/professionals/quality-patientsafety/quality-resources/tools/literacytoolkit/index.html. Accessed November 21, 2019.

10. Bass PF, Wilson JF, Griffith $\mathrm{CH}$. Shortened Instrument for Literacy Screening. J Gen Intern Med. 2003; 18(12): 1036-1038. doi: 10.1111/j.15251497.2003.10651.x. 
11. Riddle MC, Rosenstock J, Gerich J, et al. The Treat-toTarget Trial: randomized addition of glargine or human NPH insulin to oral therapy of type 2 diabetic patients. Diabetes Care. 2003; 26(11): 3080-3086. doi: 10.2337/diacare.26.11.3080.

12. Stoller WA. Individualizing insulin management. Three practical cases, rules for regimen adjustment. Postgrad Med. 2002;111(5):51-4, 59-60, 63-6. doi: 10.3810/pgm.2002.05.1197.

13. Joint Commission of Pharmacy Practitioners. Pharmacists' Patient Care Process; 2014. https://jcpp.net/wpcontent/uploads/2016/03/PatientCareProcess-withsupporting-organizations.pdf. Accessed November 21, 2019.

14. Ferguson MO, Long JA, Zhu J, et al. Low health literacy predicts misperceptions of diabetes control in patients with persistently elevated A1C. Diabetes Educ. 2015; 41(3):309-319. doi: 10.1177/0145721715572446.

15. Fan JH, Lyons SA, Goodman MS, et al. Relationship between health literacy and unintentional and intentional medication nonadherence in medically underserved patients with type 2 diabetes. Diabetes Educ. 2016; 42(2): 199-208.

16. Singh S, Acharya SD, Kamath A, et al. Health literacy status and understanding of the prescription instructions in diabetic patients. J Diabetes Res. 2018 4517243. Epub 2018. doi: 10.1155/2018/4517243.

17. White RO, Chakkalakal RJ, Presley CA, et al. Perceptions of provider communication among vulnerable patients with diabetes: influences of medical mistrust and health literacy. $J$ Health Commun. 2016; 21(2): 127-134. doi: 10.1080/10810730.2016.1207116.
18. Friis K, Vind BD, Simmons RK, et al.. The relationship between health literacy and health behaviour in people with diabetes: A Danish population-based study. J Diabetes Res. 2016:7823130. Epub 2016 Sep 28. doi: 10.1155/2016/7823130.

19. Wallace AS, Seligman HK, Davis TC, et al. Literacyappropriate educational materials and brief counseling improve diabetes self-management. Patient Educ Couns. 2009;75:328-333. doi: 10.1016/j.pec.2008.12.017.

20. Rothman RL, DeWalt DA, Malone $R$, et al. Influence of patient literacy on the effectiveness of a primary care-based diabetes disease management program. JAMA. 2004;292:1711-1716. doi: 10.1001/jama.292.14.1711.

21. Kim S, Love F, Quistberg DA, et al. Association of health literacy with self-management behavior in patients with diabetes. Diabetes Care. 2004;27:29802982. doi: 10.2337/diacare.27.12.2980.

22. Wolff K, Chambers L, Bumol S, et al. The PRIDE (Partnership to Improve Diabetes Education) Toolkit. Diabetes Educ. 2016; 42(1): 23-33. doi: $10.1177 / 0145721715620019$.

23. Protheroe J, Rathod T, Bartlam B, et al. The feasibility of health trainer improved patient self-management in patients with low health literacy and poorly controlled diabetes: a pilot randomised controlled trial. J Diabetes Res. 2016; 1-11. doi: 10.1155/2016/6903245.

24. Sugita $H$, Shinohara $R$, Yokomichi $H$, et al. Effect of text messages to improve health literacy on medication adherence in patients with type 2 diabetes mellitus: A randomized controlled pilot trial. Nagoya J Med Sci. 2017; 79:313-321. doi: 10.18999/nagjms.79.3.313. 
Table 1. Sample Teach-Back Method and Action Plan

Sample Teach-Back Method

"We covered a lot today about your diabetes and I want to make sure I explained things clearly. What are the three methods we discussed that you have to do to help control your diabetes?"

If the patient cannot remember or accurately repeat what is asked, information is clarified and the patient is asked to teach-back the information until they are able to correctly describe medical instruction.

\section{Sample Action Plan}

Goal \# 1: To keep my sugars between $80-130 \mathrm{mg} / \mathrm{dL}$

Plan: Inject 40 units of Lantus underneath the skin every day at 10 P.M.

How sure am I that I can do this?

$\begin{array}{llllllllll}1 & 2 & 3 & 4 & 5 & 6 & 7 & 8 & 9 & 10\end{array}$

Table 2. Baseline Demographics

\begin{tabular}{|c|c|c|c|}
\hline Variable $^{a}$ & $\begin{array}{l}\text { Control } \\
(\mathrm{N}=12)\end{array}$ & $\begin{array}{l}\text { Intervention } \\
(\mathrm{N}=11)\end{array}$ & P-Value \\
\hline Age & $63.2 \pm 12.7$ & $55.5 \pm 14.3$ & 0.19 \\
\hline \multicolumn{4}{|l|}{ Gender } \\
\hline Male & $5(41.7)$ & $4(36.4)$ & 0.32 \\
\hline Female & $7(58.3)$ & $7(63.6)$ & \\
\hline \multicolumn{4}{|l|}{ Race } \\
\hline Black & 11 (91.7) & $10(90.9)$ & NS \\
\hline Hispanic & $1(8.3)$ & $1(9.1)$ & \\
\hline \multicolumn{4}{|l|}{ Education } \\
\hline Some high school or less & $8(66.7)$ & $5(45.5)$ & \\
\hline High school graduate or greater & $4(33.3)$ & $5(45.5)$ & 0.67 \\
\hline Not reported & 0 & $1(10.0)$ & \\
\hline \multicolumn{4}{|l|}{ Annual Income } \\
\hline$<\$ 20,000$ & $11(91.7)$ & $9(81.8)$ & \\
\hline$\$ 20,000-40,000$ & 0 & $1(9.1)$ & NS \\
\hline Not reported & $1(8.3)$ & $1(9.1)$ & \\
\hline \multicolumn{4}{|l|}{ Diabetes Subtype } \\
\hline Type 1 & $1(8.3)$ & 0 & NS \\
\hline Type 2 & $11(91.7)$ & $11(100)$ & \\
\hline Duration of Diabetes, yrs & $19.2 \pm 11.9$ & $6.4 \pm 6.1$ & 0.01 \\
\hline REALM-R Score & $2 \pm 2.5$ & $4.1 \pm 2.2$ & 0.06 \\
\hline DNT-5 Score & $1.3 \pm 1.8$ & $1.3 \pm 1.3$ & 0.97 \\
\hline A1c, $\%$ & $9.3 \pm 1.7$ & $9.7 \pm 1.3$ & 0.47 \\
\hline
\end{tabular}

a Values are expressed as number (\%) and means \pm standard deviations

${ }^{\mathrm{b}}$ Not significant, $\mathrm{P}>0.05$

Abbreviations: REALM-R = Rapid Estimate of Adult Literacy in Medicine- Revised; DNT-5 = and Diabetic Numeracy Test-5 
Table 3. Diabetes Regimen and Insulin Dosages

\begin{tabular}{llll}
\hline Parameter & $\begin{array}{l}\text { Control } \\
(\mathrm{N}=12)\end{array}$ & $\begin{array}{l}\text { Intervention } \\
(\mathrm{N}=11)\end{array}$ & P-Value ${ }^{\mathrm{b}, \mathrm{c}}$ \\
\hline $\begin{array}{l}\text { Baseline } \\
\text { Diabetes Regimen }\end{array}$ & & \\
Insulin-Only & & & \\
Insulin- Orals & $4(33.3)$ & 0 & 0.95 \\
Orals-Only & $6(50.0)$ & $6(54.5)$ & 0.83 \\
Other & $2(16.7)$ & $4(36.4)$ & 0.95 \\
Insulin Dosage, units/kg ${ }^{b}$ & 0 & $1(9.1)$ & 0.96 \\
Basal-Only & & & \\
Bolus-Only & $0.65 \pm 0.41$ & $0.40 \pm 0.35$ & 0.14 \\
Total (basal and bolus-only) & $0.52 \pm 0.44$ & $0.06^{\mathrm{d}}$ & $\mathrm{NS}$ \\
\hline Study Termination at 3 months & $0.96 \pm 0.78$ & $0.41 \pm 0.35$ & 0.05 \\
Diabetes Regimen & & & \\
Insulin-Only & & & 0.04 \\
Insulin- Orals & & 0 & 0.51 \\
Orals-Only & $5(41.7)$ & $7(63.6)$ & 0.25 \\
Other & $6(50.0)$ & $3(27.3)$ & 0.96 \\
Insulin Dosage, units $/ \mathrm{kg}^{b}$ & $1(8.3)$ & $1(9.1)$ & \\
Basal-Only & 0 & & 0.20 \\
Bolus-Only & & $0.42 \pm 0.23$ & $\mathrm{NS}$ \\
Total (basal and bolus- only) & $0.61 \pm 0.36$ & 0.12 \\
\hline
\end{tabular}

${ }^{\text {a }}$ Values are expressed as number (\%)

${ }^{b}$ Values are expressed as means \pm standard deviation

${ }^{c} \mathrm{NS}=$ not significant, $\mathrm{P}>0.05$

${ }^{\mathrm{d}}$ Standard deviation not provided with $\mathrm{N}=1$

Table 4. Effect of Treatment on Primary and Secondary Outcomes at 3 Months

\begin{tabular}{llll}
\hline Variable & $\begin{array}{l}\text { Control } \\
(\mathrm{N}=12)\end{array}$ & $\begin{array}{l}\text { Intervention } \\
(\mathrm{N}=11)\end{array}$ & P-Value $^{\mathrm{b}}$ \\
\hline End A1c, \% & $8.2 \pm 1.9$ & $7.7 \pm 1.1$ & 0.79 \\
\hline Change in A1c (Post - Pre, \%) & $-1.0 \pm 2.2$ & $-2.0 \pm 1.3$ & 0.02 \\
\hline Change in A1c (\% Reduction) & $-9.1 \pm 17.8$ & $-20.3 \pm 11.9$ & 0.04 \\
\hline Alc < 7\%, no. & $2(9.1)$ & $2(9.1)$ & $\mathrm{NS}$ \\
\hline Blood glucose < 70 mg/dL & $0.1 \pm 0.3$ & 0 & $\mathrm{NS}$ \\
\hline Blood glucose $>180 \mathrm{mg} / \mathrm{dL}$ & $1.9 \pm 1.6$ & $1.7 \pm 1.2$ & 0.97 \\
\hline Hypoglycemic events/week, no. & $0.8 \pm 1.0$ & $0.2 \pm 0.2$ & 0.08 \\
\hline Hyperglycemic events/week, no. & $2.1 \pm 3.8$ & $0.1 \pm 0.2$ & 0.04 \\
\hline Hospitalizations, no. & $0.2 \pm 0.6$ & 0 & $\mathrm{NS}$ \\
\hline Emergency room visits, no. & $0.4 \pm 1.2$ & $0.1 \pm 0.3$ & $\mathrm{NS}$ \\
\hline Testing per day, no. & $2.2 \pm 1.3$ & $1.5 \pm 1.0$ & 0.18 \\
\hline Medication adherence rate, \% & $78.0 \pm 13.8$ & $87.7 \pm 11.7$ & $\mathrm{NS}$ \\
\hline
\end{tabular}

a Values are expressed as number (\%) and means \pm standard deviations

${ }^{\mathrm{b}}$ Not significant, $\mathrm{P}>0.05$ 
Figure 1. Enrollment, randomization, and follow-up

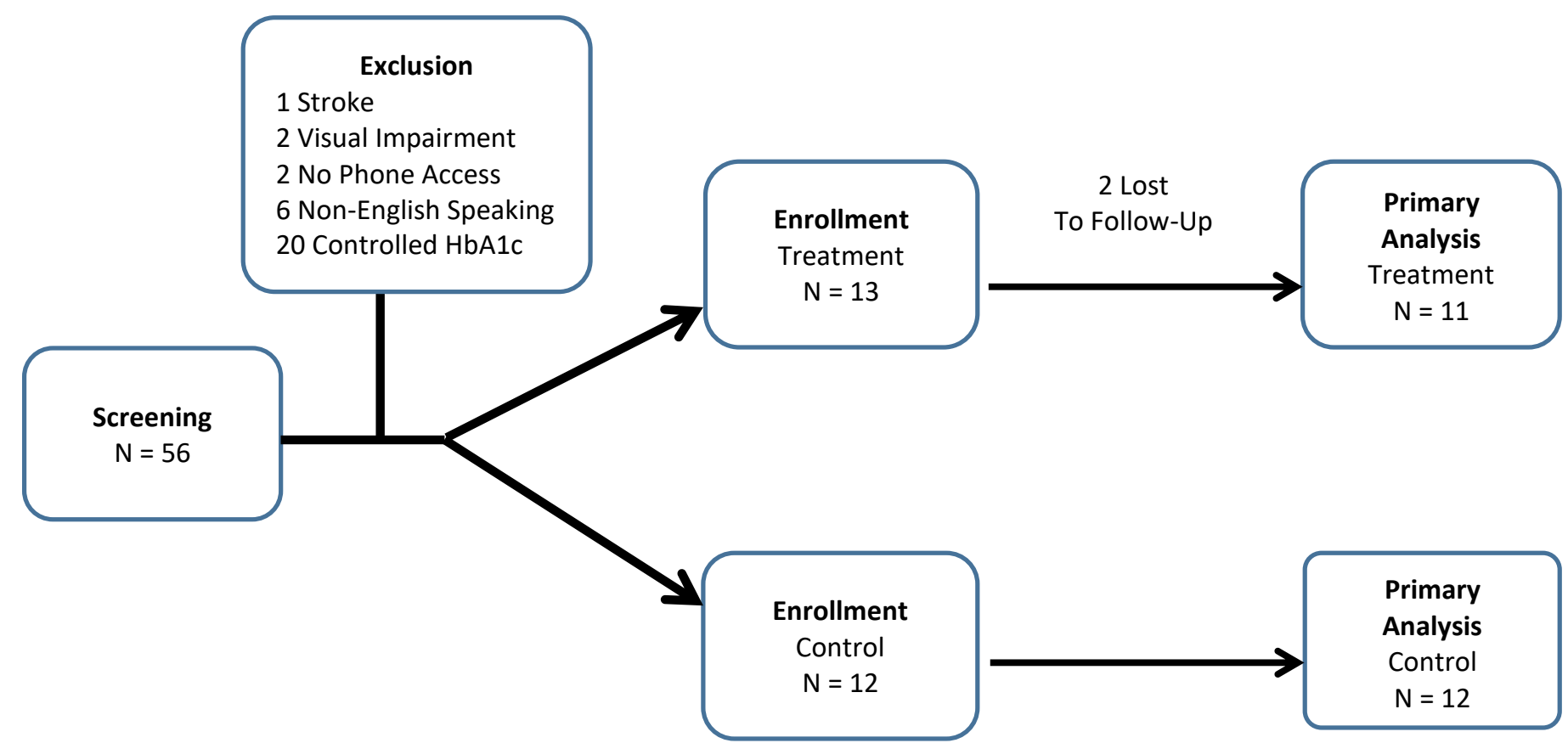


Figure 2. Change in HgbA1c for treatment and usual care group for each participant from baseline*

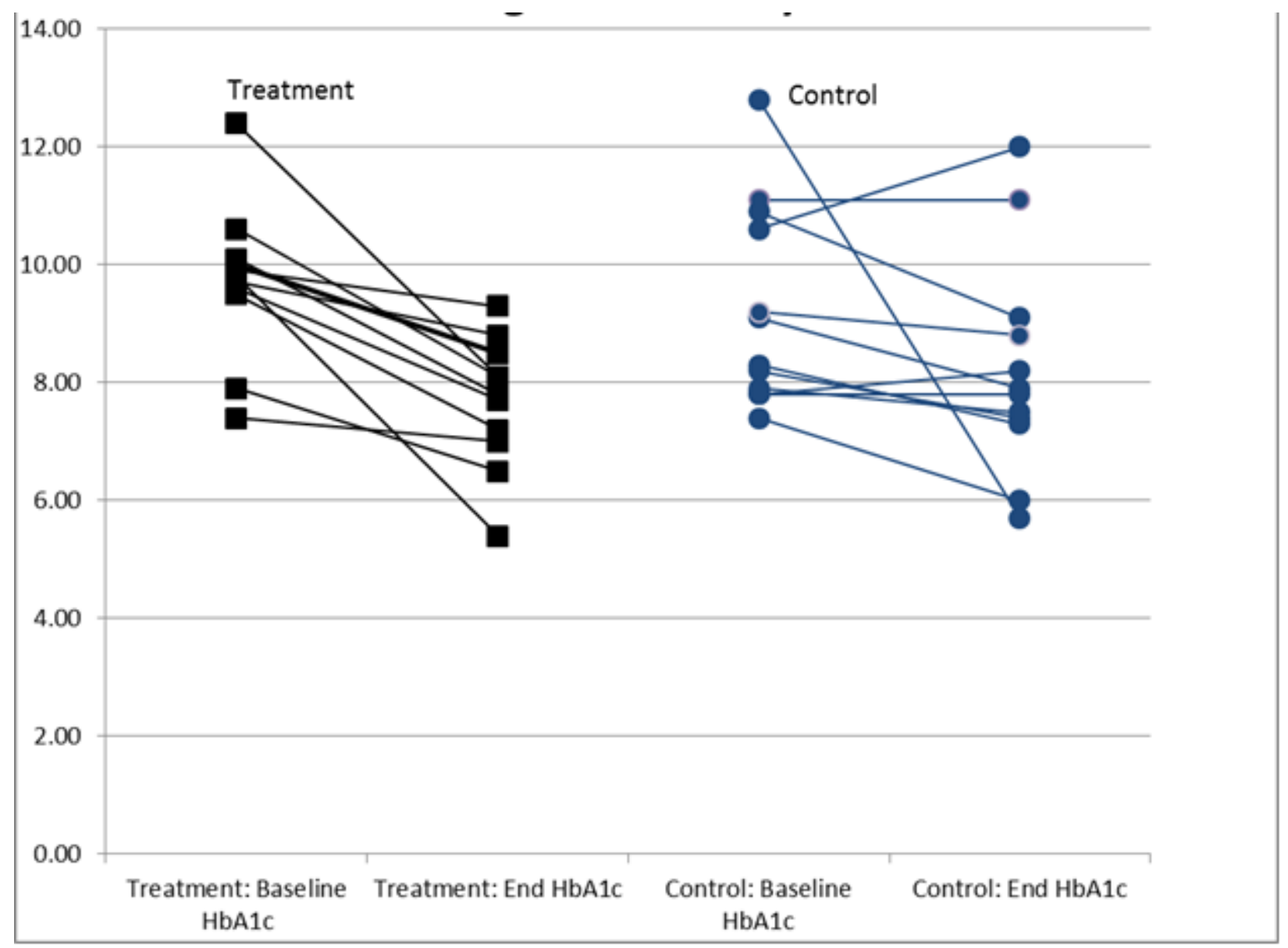

$* \mathrm{P}=0.02$ 